\title{
ROLLING STONES WITH NONCONVEX SIDES II: ALL TIME REGULARITY OF INTERFACE AND SURFACE
}

\author{
Ki-Ahm Lee and Eunjai Rhee
}

\begin{abstract}
In this paper we consider the evolution of the rolling stone with a rotationally symmetric nonconvex compact initial surface $\Sigma_{0}$ under the Gauss curvature flow. Let $X: S^{n} \times[0, \infty) \rightarrow \mathbb{R}^{n+1}$ be the embeddings of the sphere in $\mathbb{R}^{n+1}$ such that $\Sigma(t)=X\left(S^{n}, t\right)$ is the surface at time $t$ and $\Sigma(0)=\Sigma_{0}$. As a consequence the parabolic equation describing the motion of the hypersurface becomes degenerate on the interface separating the nonconvex part from the strictly convex side, since one of the curvature will be zero on the interface. By expressing the strictly convex part of the surface near the interface as a graph of a function $z=f(r, t)$ and the non-convex part of the surface near the interface as a graph of a function $z=\varphi(r)$, we show that if at time $t=0, g=\frac{1}{n} f_{r}^{n-1}$ vanishes linearly at the interface, the $g(r, t)$ will become smooth up to the interface for long time before focusing.
\end{abstract}

\section{Introduction}

In this work, we are going to consider the wearing process of a rolling stone on a plane. In the figure 1 , since the collision of a rolling stone on the plane causes the erosion of the surface, the speed of the erosion is proportional to the number of outward normal directions on a given surface area element, namely the Gauss curvature of the convex surface. Let us denote $\Sigma$ be the surface evolving by the Gauss curvature flow and $\Sigma^{*}$ be the convex envelope of $\Sigma$ which is the smallest convex surface containing $\Sigma$. Then any point $P$ on the rolling stone $\Sigma$ will propagate with the speed of $\Sigma$,

$$
\frac{\partial P}{\partial t}=K_{+}^{*} N
$$

in the inward normal direction $N$, where $K_{+}^{*}$ is the Gauss curvature of $\Sigma^{*}$ for $P \in \Sigma \cap \Sigma^{*}$ and otherwise zero. We denote by $g_{i j}$ the metric and a second fundamental form of $\Sigma$ and by $g_{i j}$ and $h_{i j}$. We also denote the inverse of $g_{i j}$

Received October 23, 2010.

2010 Mathematics Subject Classification. 35K20.

Key words and phrases. free boundary problems, degenerate fully nonlinear equations, Gauss curvature flow. 
and $h_{i j}$ by $g^{i j}$ and $\left(h^{-1}\right)^{i j}$. The Weingarten map is given by

$$
h_{i}^{j}=g^{j k} h_{k i}
$$

and the eigenvalues, $\lambda_{1}, \ldots, \lambda_{n}$, of $h_{i}^{j}$ are called principle curvatures. The Gauss curvature flow was introduced by Firey [14] and he showed that the smooth compact, strictly convex hypersurfaces with some symmetry shrinks to a round point. Tso [21] showed that the Gauss curvature flow with strictly convex initial surface will be smooth up to a vanishing time.

Daskalopoulos and Hamilton considered the case when the initial surface has a flat side. At the interface of the flat side and the strictly convex side, the curvature becomes degenerate which behaves like a free boundary with finite speed of propagation. In [5], they proved the short time existence and smooth regularity of the surface based on a Schauder theory of a degenerate equation. Andrews [1] showed that the $C^{1,1}$ regularity of a viscosity solution before a vanishing time of the surface. Under the same assumption of [5], Daskalopoulos and Lee, $[11]$, showed that the strictly convex side remains smooth up to the focusing time of the flat side and that the optimal regularity will be $C^{2, \alpha}$ for some $0<\alpha<1$ when the surface is radially symmetric. They also extended these results for any two dimensional surface. Main difference in two dimension is that the equation is fully nonlinear while it is semilinear in one dimension and that the equation is degenerate when the some second derivatives are degenerate. It is noticeable that the porous medium equation is degenerate when the density is zero and that the $p$-Laplace equation is degenerate when the gradient of the density is zero.

Ishii and Mikami, [16], [17], showed the existence of a viscosity solution to the case when the surface is not necessarily convex through the level set approach.

We will consider the case where the initial radial symmetric surface has a non-convex side and as a result the parabolic equation becomes degenerate along the interface of the non-convex surface and the convex surface. In this paper, we discuss the existence and regularity of the solution, and regularity of the free boundary.

Let us assume that initially we have surface

$$
\Sigma=\Sigma_{0} \cup \Sigma_{1},
$$

where $\Sigma_{0}$ is the non-convex side and $\Sigma_{1}$ is the strictly convex part of the surface, $\Sigma$. The junction between the two sides is the $(n-1)$-dimensional surface

$$
\Gamma=\Sigma_{0} \cap \Sigma_{1} .
$$

Now we assume $\Sigma_{0}$ is a concave graph $z=\varphi(x)$ over a hyper plane.

Since the equation is invariant under the rotation, we may assume the hyper plane is $z=0$ plane and that $\Sigma_{1}$ lies above this plane. The the lower part of 
$\Sigma$ can be written as the graph of a function

$$
z=f(x)
$$

over a compact domain $\Omega \subset \mathbb{R}^{n}$ on which the non-convex part can be written as a graph $z=\varphi(x)$. Suppose $z=f^{*}(x)$ is the convex envelope of the non-convex surface $z=f(x)$. We can choose the domain $\Omega$ to be the set

$$
\Omega=\left\{x \in \mathbb{R}^{n}:\left|\nabla f^{*}\right|(x)<\infty\right\}
$$

so that $f^{*}$ turns vertical at the boundary $\Gamma$. Let us denote by $T_{c}$ the time when the area of the non-convex side $\Sigma_{o}$ of the surface shrinks to zero. Since we only consider the surface symmetric with respect to $z$-axis, we may denote the lower part of $\Sigma_{1}$ by $z=f(r, t)$ for $|x|=r$ and the non-convex part $\Sigma_{0}$ by $z=\varphi(r)$ for $|x|=r$. Note that $f(r, 0)=\varphi(r)$ on $\Sigma_{0}$. Suppose $z=f^{*}(r, t)$ be the convex envelope of the non-convex surface $z=f(r, t)$. Then under the Gauss curvature flow, the envelope evolves as

$$
f_{t}=\frac{\operatorname{det}\left(D^{2} f^{*}\right)}{\left(1+\left|D f^{*}\right|^{2}\right)^{\frac{n+1}{2}}} .
$$

Let $\Omega(f)=\left\{(x, t):|x|=r, f(r, t)=f^{*}(r, t)\right\}$ and $\Omega_{t}(f)=\{x:(x, t) \in$ $\Omega(f)\}$. The free boundary is denoted by $\Gamma(f)=\partial \Omega(f)$ and $\Gamma_{t}(f)=\{x$ : $(x, t) \in \Gamma(f)\}$. In particular, we denote $\Omega_{t}=\Omega_{t}(f)$ and $\Gamma_{t}=\Gamma_{t}(f)$.

Conditions on $\Omega(f)=\left\{r: f(r, t)=f^{*}(r, t)\right\}$ :

- $\max \varphi<\max f$ on $[0, T]$

- $f_{r}=0$ on $\Gamma_{0}$

- $0<\delta<\left|\left(f_{r}\right)^{n-1} f_{r r}\right|<C$ on $\Gamma_{0}$

- $z=\varphi(r)$ is a linear function. In the other words, the surface $\{(x, z)$ : $\left.z=\varphi(|x|), x \in \mathbb{R}^{n}\right\}$ is a cone in $\mathbb{R}^{n+1}$.

The first condition is for avoiding singularities that could appear by the surface on the top wearing out faster than the non-convex part vanishing. And the second condition is imposed for finite speed of propagation which will be explained in the following paragraph. $\varphi$ can be a general concave function in the forth condition.

To understand the local behavior, we consider a simple model near the free boundary $r=\gamma(t)$. (1.1) will be

$$
f_{t}=\frac{f_{r r}^{*}}{I^{n+1}}\left(\frac{f_{r}^{*}}{r}\right)^{n-1}
$$

where $I=\left(1+\left(f_{r}^{*}\right)^{2}\right)^{\frac{1}{2}}$. Now we want to investigate whether the speed of propagation of the free boundary is non-degenerate and finite as it does in the flat spot case, [11]. $f_{r}$ will be zero on the free boundary otherwise it will propagate with infinite speed. Notice that on the free boundary $\Gamma(f)$, we have

$$
f^{*}(\gamma(t), t)=\varphi(\gamma(t), t)
$$


Then

$$
\begin{gathered}
f_{t}^{*}+f_{r}^{*} \gamma^{\prime}(t)=\varphi_{r} \gamma^{\prime}(t), \\
\gamma^{\prime}(t)=\frac{f_{t}^{*}}{\varphi_{r}-f_{r}^{*}},
\end{gathered}
$$

and $f_{t}^{*}=f_{t}$ on $\Omega(t)$. These imply

$$
\gamma^{\prime}(t)=\frac{\left(f_{r}^{*}\right)^{n-1} f_{r r}^{*}}{r^{n-1}\left(\varphi_{r}-f_{r}^{*}\right) I^{n+1}}
$$

$f_{r}=0$ otherwise it will propagate with infinite speed. If $\lim _{r \rightarrow \gamma(t)}+f_{r}(x, t)>0$, then we have $f_{r r}^{*}(\gamma(t), t)=\infty$ which implies the speed of the propagation of the free boundary is $\infty$. If we expect the speed of the free boundary to be finite, $f_{r}^{*}(\gamma(t), t)=\lim _{r \rightarrow \gamma(t)}+f_{r}(x, t)=0$.

To find the behavior of $f_{r}$ away from the free boundary, let us try $f_{r} \approx$ $(r-\gamma(t))^{\alpha_{0}}$ for some $\alpha_{o}$. From the fact that $\gamma^{\prime}(t), \varphi_{r}(\gamma(t))$, and $I$ are of order one, it is easy to see $\alpha_{o}=\frac{1}{n}$ and that we expect the optimal regularity of $f$ to be $C^{1, \frac{1}{n}}$. However, if we let the pressure $g(r, t)=\frac{1}{n} f_{r}^{n} \approx(r-\gamma(t))$,

$$
g_{t}=\frac{g^{\alpha} g_{r r}}{r^{n-1} I^{n+1}}-(n-1) \frac{g^{\alpha} g_{r}}{r^{n} I^{n+1}}-(n+1) \frac{g^{\frac{1}{n}} g_{r}^{2}}{r^{n-1} I^{n+3}},
$$

where $\alpha=\frac{n-1}{n}, I=\left(1+(n g)^{\frac{2}{n}}\right)^{\frac{1}{2}}$ and we may expect better regularity for $g$ as for the pressure of the porous medium equation [5]. Let us return back to the original equation with free boundary condition

$$
\gamma^{\prime}(t)=\frac{f_{r r}^{*}}{\varphi_{r}}\left(\frac{f_{r}^{*}}{r}\right)^{n-1} \quad(r, t) \in \Gamma(f)
$$

since $I=\left(1+f_{r}^{2}\right)^{(n+1) / 2}=1$ on $\Gamma(f)$. Since $g(\gamma(t), t)=0$,

$$
\gamma^{\prime}(t)=-\frac{g_{t}}{g_{r}}
$$

With (1.2), we conclude

$$
g_{t}=-\frac{g_{r}^{2}}{\varphi_{r} r^{n-1}}
$$

In this paper, we discuss the existence and regularity of the solution $g$ satisfying

$(\mathrm{GCFP}) \begin{cases}g_{t}=\frac{1}{I^{n+1}}\left\{\frac{g^{\alpha} g_{r r}}{r^{n-1}}-(n-1) \frac{g^{\alpha} g_{r}}{r^{n}}-(n+1) \frac{g^{\frac{1}{n}} g_{r}^{2}}{r^{n-1} I^{n+3}}\right\} & \text { in } \Omega(g) \\ g_{t}(r, t)=-\frac{g_{r}^{2}}{\varphi_{r} r^{n-1}} & \text { on } \Gamma(g)\end{cases}$

and regularity of the free boundary $\Gamma_{t}=\partial\{x: g(r, t)=0\}$ with finite speed of propagation where $\alpha=\frac{n-1}{n}$ and $I=\left(1+(n g)^{\frac{2}{n}}\right)^{\frac{n+1}{2}}$.

The first result shows the short time existence and the free boundary is smooth for short time: 
Theorem 1.1 (The Short Time Existence). If the functions $g^{0}, g_{r}^{0}$ and $g^{\alpha} g_{r r}^{0}$, restricted to the compact domain $\Omega_{0}$, extend continuously up to the boundary $\Omega_{0}$, with extensions which are Hölder continuous on $\Omega_{0}$ of class $C^{\gamma}\left(\Omega_{0}\right)$, for some $\gamma>0$, and $g_{r}^{0} \neq 0$ along $\Gamma_{0}$, then there exists a number $T>0$ for which the Gauss curvature flows, (GCF), admits a solution $\Sigma(t)$ whose strictly convex side $\Sigma_{0}(t)$ is smooth up to the interface $\Gamma_{t}$, when $0<t<T$. In particular, the free boundary $\Gamma$ is a smooth surface in the space and time, when $0<t<T$.

The second theorem shows $g$ remains smooth up to the free boundary till the non-convex part focuses to a point at the time $T_{c}$ :

Theorem 1.2 (The Long Time Existence). Under the assumption of the Theorem 1.1, there exists a solution $\Sigma(t)$ of $(\mathbf{G C F})$ whose pressure-like function $g$ is smooth up to the free boundary $\Gamma$ for all $t<T_{c}$ where $T_{c}$ is the focusing time of the non-convex surface. In particular the interface $\Gamma_{t}$ is smooth in $t$ for $0<t<T_{c}$.

Inspired by [5], [10], [11], the proof of Theorem 1.1 is based on the idea of global change of coordinates by setting $g(h(z, t), t)=z$ where $\{x: x=h(z, t)\}$ is the level set of $g$. This transformation enables us to change the free boundary problem into an fixed boundary value problem

$$
\begin{cases}M(h)=h_{t}-F\left(t, z, z^{\alpha} h_{z z}\right) & \text { in }\{0<z \leq 1\} \times[0, T] \\ h_{t}(z, t)=H\left(h_{z}(z, t), h\right) & \text { on }\{z=0\} .\end{cases}
$$

This equation is governed by metric $s$ where

and

$$
d^{2} s=\frac{d x^{2}}{2 x^{\alpha}}
$$

$$
\Delta_{s} h=x^{\alpha} h_{x x}
$$

which is no longer degenerating with respect to this new metric $s$. We employ Schauder estimate with respect to $s$ for the model equation [20]. To prove the existence of solution at Section 2, we apply the inverse function theorem between certain Banach spaces. Section 3 will be devoted to our second result, the long time existence up to the free boundary till the non-convex part vanishes and $C^{\infty}$ smoothness of the free boundary. First let us summarize the notations.

\section{Notations:}

(1) The convex surface $\Sigma=\Sigma_{0} \cup \Sigma_{1}$ where $\Sigma_{0}$ is the non-convex side and $\Sigma_{1}$ is the strictly convex part of the surface, $\Sigma$.

(2) $f^{*}$ is the convex envelope of $f$ which the supremum of all linear functions below $f$.

(3) The domains will be defined as the followings:

$$
\begin{aligned}
& \Omega(t)=\left\{x \in \mathbb{R}^{n}:\left|D f^{*}(x, t)\right|<\infty\right\} \\
& \Omega(g)=\{(x, t): x \in \Omega(t), 0 \leq t<T, g(x, t)>0\} \\
& \Omega_{t}=\{x \in \Omega(t): g(x, t)>0\}
\end{aligned}
$$


$\Omega_{t}^{k}=\{x \in \Omega(t): 0<g(x, t) \leq k\}$,

$\Omega_{t} \times[0, T]=\cup_{0 \leq t \leq T}\left(\Omega_{t} \times\{t\}\right)=\Omega(g)$,

$\Omega_{t}^{k} \times[0, T]=\cup_{0 \leq t \leq T}\left(\Omega_{t}^{k} \times\{t\}\right)$,

$\Gamma(g)=\partial\{(x, t): g(x, t)=0\}, \Gamma_{t}=\{x:(x, t) \in \Gamma(g)\}=\partial\{x: g(x, t)=$ $0\}$ and $\Gamma_{t}$ is the graph of $r=\gamma(t)$,

$S_{0}=\{x>0\}$ and $S=S_{0} \times[0, \infty)$.

Notice that $\Omega(t)=\{x: g(x, t)=0\} \cup \Omega_{t}$ and $\Omega(0)=\Omega$.

$Q_{R}^{+}=\left\{(x, t): 0 \leq x \leq R, 1-R^{2-\alpha} \leq t \leq 1\right\}$.

(4) The parabolic distance between two points $P=\left(x_{1}, t_{1}\right)$ and $Q=$ $\left(x_{2}, t_{2}\right)$ is

$$
s[P, Q]=\frac{\left|x_{1}-x_{2}\right|}{\left|x_{1}^{\frac{\alpha}{2}}+x_{2}^{\frac{\alpha}{2}}\right|}+\sqrt{\left|t_{1}-t_{2}\right|} .
$$

(5) $D_{x} f=f_{x}$ and $\bar{D}_{x} f=x^{\alpha} f_{x}$.

$D_{x}^{2 k} f=\left(\bar{D}_{x} D_{x}\right)^{k} f$ and $D_{x}^{2 k+1} f=D_{x}\left(\bar{D}_{x} D_{x}\right)^{k} f$.

(6) The Hölder norms of $f$ in a set $A$ :

$$
\begin{aligned}
& \|f\|_{C_{s}^{0}(A)}=\sup _{x \in A}|f(x)|,\|f\|_{H_{s}^{\gamma}(A)}=\sup _{P \neq Q \in A} \frac{|f(P)-f(Q)|}{s(P, Q)^{\gamma}}, \\
& \|f\|_{H_{s}^{2+\gamma}(A)}=\left\|x^{\alpha} f_{x x}\right\|_{H_{s}^{\gamma}(A)}+\left\|f_{t}\right\|_{H_{s}^{\gamma}(A)} \text {, } \\
& \|f\|_{C_{s}^{2}(A)}=\|f\|_{C_{s}^{0}(A)}+\left\|f_{x}\right\|_{C_{s}^{0}(A)}+\left\|x^{\alpha} f_{x x}\right\|_{C_{s}^{0}(A)}++\left\|f_{t}\right\|_{C_{s}^{0}(A)}, \\
& \|f\|_{C_{s}^{2 k}(A)}=\sum_{i+j \leq k}\left\|\bar{D}_{x, x}^{i} D_{t}^{j} f\right\|_{C_{s}^{0}(A)}, \\
& \|f\|_{H_{s}^{k+\gamma}(A)}=\sum_{2 i+j=k}\left\|D_{x}^{i} D_{t}^{j} f\right\|_{H_{s}^{\gamma}(A)},\|f\|_{C_{s}^{2 k+\gamma}(A)}=\|f\|_{C_{s}^{2 k}(A)}+ \\
& \|f\|_{H_{s}^{2 k+\gamma}(A)} \text {. } \\
& \text { (7) } T_{0} g(x, t)=g(0,1) \text {, } \\
& T_{1} f(x, t)=f(0,1)+f_{x}(0,1) x, \\
& T_{2-\alpha, 1} f(x, t)=f(0,1)+f_{x}(0,1) x+\frac{1}{(2-\alpha)(1-\alpha)} x^{\alpha} f_{x x}(0,1) x^{2-\alpha}+f_{t}(0,1)(t-1) \text {, } \\
& R_{i} f=f-T_{i} f \text { for } i=0,1 \text { and } R_{2-\alpha, 1} f=f-T_{2-\alpha, 1} f .
\end{aligned}
$$

Let us start with showing the existence of the solution for short time by introducing a simple model equation in the following section.

\section{The free boundary for the Gauss curvature flow}

\subsection{The result}

In this part of the paper we will apply the results of Section 4, [20], to study the regularity of the free boundary for the Gauss curvature flow

$$
f_{t}=\frac{\left(f_{r}\right)^{n-1} f_{r r}}{r^{n-1}\left(1+f_{r}^{2}\right)^{(n+1) / 2}} .
$$

In the introduction, we show the pressure

$$
g(r, t)=\frac{1}{n} f_{r}^{n},
$$


satisfies

$$
g_{t}=\frac{1}{I^{n+1}}\left\{\frac{g^{\alpha} g_{r r}}{r^{n-1}}-(n-1) \frac{g^{\alpha} g_{r}}{r^{n}}-(n+1) \frac{g^{\frac{1}{n}} g_{r}^{2}}{r^{n-1} I^{n+3}}\right\}
$$

with $I=\left\{1+(n g)^{2 / n}\right\}^{1 / 2}$ and $\alpha=\frac{n-1}{n}$. And on the free boundary $\Gamma(t)$, we have $g\left(\Omega_{t}, t\right)=0$.

Let $\Omega$ be a compact domain in $\mathbb{R}^{n}$ and $g^{0}$ a function on $\Omega$ with $g^{0}=0$ at $\Gamma$ and $g^{0}>0$ in the interior of $\Omega$. We will study the free-boundary problem, (GCFP), for some $T>0$, where $\Omega_{t}$ is the closure of the set $\{r \in \mathbb{R}: g(r, t)>$ $0\}$. Throughout the rest of the paper we will denote by $\Omega_{t} \times[0, T]$ the set $\Omega_{t} \times[0, T]=\cup_{0 \leq t \leq T}\left(\Omega_{t} \times\{t\}\right)$. Our main result is the following regularity theorem.

Theorem 2.1. Let $\Omega$ be a compact domain in $\mathbb{R}$ and let $g^{0}$ be a function in the space $C_{s}^{2+\gamma}(\Omega)$, for some $0<\gamma<1$, with $g^{0}=0$ at $\Gamma$ and $g^{0}>0$ in the interior of $\Omega$. Moreover, assume that

$$
D g^{0}(r) \neq 0, \quad \forall r \in \Omega .
$$

Then, there exists a number $T>0$, for which the free-boundary problem, (GCFP), admits a solution $g$ whose $\frac{\partial g}{\partial t}$ is $C_{s}^{2+\gamma}$ and $\frac{\partial g}{\partial r}$ is $\tilde{C}_{s}^{2+\gamma}$ to the free boundary $\Gamma_{t} \times(0, T]$. In particular, the interface $\Gamma_{t} \times(0, T]$ is smooth.

Theorem 1.1 in the introduction is an immediate consequence of Theorem 2.1 , since $C^{\gamma}(\Omega) \subset C_{s}^{\gamma}(\Omega)$ for all $\gamma>0$. Notice that the solution of the free boundary problem in Theorem 2.1 , extended to be equal to zero on $\left(\Omega \backslash \Omega_{t}\right) \times$ $[0, T]$, is the unique weak solution of the free boundary problem, (GCFP).

To motivate the proof of the regularity Theorem 2.1 we will first compute the transformation of (GCFP), when one exchanges dependent and independent variables near the boundary. This change of coordinates converts the free boundary into a fixed boundary. Assume that the function $g$ belongs to the space $C_{s}^{2+\gamma}\left(\Omega_{t} \times[0, T]\right)$. Pick a point $P_{0}=\left(r_{0}, t_{0}\right)$ at the free boundary $\Gamma_{t} \times$ $(0, T]$. We can assume that $g_{r}\left(P_{0}\right)>0, g_{t}\left(P_{0}\right)=c$. For a positive number $\delta$ sufficiently small, we denote by $Q_{\delta}$ the cube

$$
\left|r-r_{0}\right| \leq \delta, \quad-\delta \leq t-t_{0} \leq 0
$$

and by $\Lambda_{\delta}$, the intersection

$$
\Lambda_{\delta}=\left(\Omega_{t} \times[0, T]\right) \cap Q_{\delta} .
$$

Since the first derivatives of $g$ are Hölder continuous up to the free boundary, there exists a number $\delta>0$ such that

$$
g_{r}(r, t)>0, \quad g_{t}(r, t)>0 \quad \forall(r, t) \in \Lambda_{\delta} .
$$

It follows from the Implicit Function Theorem that if the number $\delta$ is sufficiently small, we can solve the equation $z=g(r, t)$ for $(r, t) \in \Lambda_{\delta}$ with respect to $r$, yielding to a map $r=h(z, t)$ defined for all $(z, t)$ sufficiently close to the point 
$\left(0, t_{0}\right)$. Also, after a moment of thought we can see that there exists a number $\eta>0$ sufficiently small, so that $h$ is defined on the cube $B_{\eta}=\{0 \leq z \leq$ $\left.\eta, \quad-\eta \leq t-t_{0} \leq 0\right\}$.

We wish to find the differential equation satisfied by the function $h$ in $B_{\eta}$. Notice that the free boundary $g=0$ has now been transformed into the fixed boundary $z=0$. Via this change of coordinates, (GCFP) transforms into the equation

$$
\begin{aligned}
& M(h)=h_{t}-z^{\alpha} \frac{1}{J} \frac{h_{z z}}{h^{n-1} h_{z}^{2}}+(n-1) \frac{1}{J} \frac{z^{\alpha}}{h^{n}}=0, \\
& B(h)=h_{t}-\frac{1}{\varphi_{r}(h) h^{n-1} h_{z}}=0 .
\end{aligned}
$$

The operator $M$ defined above is a quasi-linear operator which becomes degenerate when $z=0$. We can easily compute its linearization $D M(h)$ at the point $h$ :

$$
\begin{aligned}
D M(h)(\tilde{h}) & =\tilde{h}_{t}-z^{\alpha} \frac{1}{J} \frac{1}{h h_{z}^{2}} \tilde{h}_{z z}+\frac{1}{J} \frac{2 z^{\alpha} h_{z z}}{h h_{z}^{3}} \tilde{h}_{z}+\frac{n-1}{J}\left\{\frac{z^{\alpha} h_{z z}}{h^{n} h_{z}^{2}}+\frac{n z^{\alpha}}{h^{n+1}}\right\} \tilde{h}, \\
D B(h)(\tilde{h}) & =\tilde{h}_{t}+\frac{\tilde{h}_{z}}{\varphi_{r}(h) h^{n-1} h_{z}^{2}}+\frac{n \tilde{h}}{\varphi_{r}(h) h^{n} h_{z}} .
\end{aligned}
$$

Since $h \in C_{s}^{2+\gamma}\left(B_{\eta}\right)$ and on the set $B_{\eta}$ we have $h_{z}(z, t)>0$ the linearized operator $D M(h)$ on $B_{\eta}$ belongs to the class of the degenerate operators studied in Section 3, [20].

Our goal is to use the results in Section 3, [20], for the proof of Theorem 2.1. However, the change of coordinates presented here is only local and can't be used directly for the proof of Theorem 2.1. In the next section we will introduce a more subtle, global change of coordinates which is based on similar ideas.

\subsection{Partial regularity}

Let $\Omega$ be a compact domain in $\mathbb{R}$ and $g^{0}$ a function on $\Omega$ with $g^{0}=0$ at $\Gamma$ and $g^{0}>0$ in the interior of $\Omega$. Assume that $g^{0} \in C_{s}^{k, 2+\gamma}(\Omega)$ for some nonnegative integer $k$ and that

$$
\left|D g^{0}(r)\right|+g^{0}(r) \geq c>0 \quad \forall r \in \Omega
$$

for some fixed positive number $c$. Denote by $D$ the unit disk $D=\{u \in \mathbb{R}$ : $|u| \leq 1\}$ and pick a smooth surface $S$, sufficiently close to the surface $z=g^{0}(r)$. Let $S: D \rightarrow \mathbb{R}^{n}$ be a smooth parametrization for the surface $S$ which maps $\partial D$ onto $S \cap\{z=0\}$. Also, let

$$
T=\left(\begin{array}{l}
T_{1} \\
T_{2}
\end{array}\right)
$$

be a smooth vector field, transverse to the surface $S$. Since $\left|D g^{0}\right| \geq c$ along $\Gamma$ and $S$ is sufficiently close to the surface $z=g^{0}(r)$, we can choose $T$ to be parallel to the plane $z=0$ in a small neighborhood of $\partial D$. In other words, 
there exists a number $\delta>0$ depending on $k$ such that $T_{2}(u)=0$ on $D \backslash D_{1-\delta}$ with $D_{1-\delta}=\{u \in \mathbb{R}:|u| \leq 1-\delta\}$.

For $\eta>0$ sufficiently small, we define the change of spatial coordinates $\Phi: D \times[-\eta, \eta] \rightarrow \mathbb{R}^{2}$ by

$$
\left(\begin{array}{l}
r \\
z
\end{array}\right)=\Phi\left(\begin{array}{l}
u \\
w
\end{array}\right)=S(u)+w T(u)
$$

The map $\Phi$ defines $r$ and $z$ as functions of the new coordinates $u$ and $w$.

Assume that $z=g(r, t)$ satisfies the initial free-boundary problem, (GCFP), where $\Omega_{t}$ is the closure of the set $\{r \in \mathbb{R}: g(r, t)>0\}$. Under the coordinate change $\Phi$, the initial data $g^{0}(r)$ transforms to a function $w^{0}(u)$ which can be made arbitrarily small, by choosing the smooth surface $S$ sufficiently close to the surface $z=g^{0}(r)$. We will see that $w^{0} \in C_{s}^{k, 2+\gamma}(D)$, since $g \in C_{s}^{k, 2+\gamma}(\Omega)$.

When $z$ evolves as a function of $(r, t)$ then, through this coordinate change $w$ evolves as a function of $(u, t)$ with $u \in D$. Notice that by our choice of the parametrization $S$ we have $u \in \partial D$ if and only if $z=0$. Hence the freeboundary where $z=g(r, t)=0$ is mapped onto the fixed lateral boundary of the cylinder $D \times[0, T]$.

The evolution of $w$ is described in the following theorem:

Theorem 2.2. Let $\Omega$ be a compact domain in $\mathbb{R}, k$ a nonnegative integer and let $g^{0}$ be a function in the space $C_{s}^{k, 2+\gamma}(\Omega)$ for some number $\gamma$ in $0<\gamma<1$. Assume that $g^{0}>0$ in the interior of $\Omega$ and $g^{0}=0$ at $\Gamma$ with

$$
\left|D g^{0}(r)\right|+g^{0}(r) \geq c \quad \forall r \in \Omega .
$$

Then, under the coordinate change, (GCFP) converts into the initial value problem

with

$$
\begin{cases}M w & =0 \quad(u, t) \in D \times[0, T] \\ B w & =0 \quad(u, t) \in \partial_{0} D \times[0, T] \\ w(u, 0) & =w^{0}(u) \quad u \in D\end{cases}
$$

$$
\left\{\begin{array}{l}
M w=w_{t}-\left(u^{\alpha} F(t, u, w, D w) w_{u u}+G(t, u, w, D w)\right) \\
B w=H(t, u, w, D w)
\end{array}\right.
$$

and $w^{0} \in C_{s}^{k, 2+\gamma}(D)$. Moreover, if $T \leq \tau_{k}$, with $\tau_{k}$ sufficiently small depending on $c$ and $k$, the operator $M$ satisfies all the hypotheses of Theorem 4.4, [20].

Proof. We choose a sufficiently small number $\delta>0$, such that

$$
T_{2}(u)=0 \quad \text { on } D \backslash D_{1-\delta}
$$

with $T=\left(\begin{array}{c}T_{1} \\ T_{2}\end{array}\right)$ denoting, as above, the transverse vector field to the surface $S$. Notice that by choosing the smooth surface sufficiently close to the surface $z=g^{0}(r)$, we can make $\delta$ to depend only on the constant $c$.

To show that $w$ satisfies an equation of the desired form in the interior cylinder $D_{1-\delta} \times[0, T]$ is straight forward. Hence, we will restrict our attention 
to $D \backslash D_{1-\delta}$. We start by expressing the first and second derivatives of $z$ with respect to $r, t$ in terms of the first and second derivatives of $w$ with respect to $u, t$.

After some calculations, we see that $z=g(r, t)$ evolves as

$$
g_{t}=\frac{1}{I^{n+1}}\left\{\frac{g^{\alpha} g_{r r}}{r^{n-1}}-(n-1) \frac{g^{\alpha} g_{r}}{r^{n}}-(n+1) \frac{g^{\frac{1}{n}} g_{r}^{2}}{r^{n-1} I^{n+3}}\right\}
$$

and that $w$ evolves as

$\frac{\partial w}{\partial t}=\frac{z^{\alpha}}{r^{n-1} I^{n+1}}\left\{\frac{\partial^{2} w}{\partial u^{2}}+\frac{r_{u w}+2 r_{u w}}{r_{w}}\left(\frac{\partial w}{\partial u}\right)^{2}+\frac{r_{u u}}{r_{w}}-\frac{z_{u u}}{r_{u} r_{w} z_{u}}\right\}+(n-1) \frac{z^{\alpha}}{I^{n+1} r^{n}}$.

Notice that on $D \backslash D_{\delta}$ the $z$ coordinate is independent of $w\left(T_{2}=0\right)$. In other words $z=S_{2}$, with $S_{2}$ denoting the $z$ coordinate of the parametrization $S$. Therefore, since $S_{2}$ maps $\partial D$ onto $z=0, z=x(u)$ on $D \backslash D_{\delta}$, strictly positive in the interior of $D$ and such that

$$
\vartheta(u) \sim d(u) \quad \text { on } \quad D \backslash D_{1-\delta}
$$

with $d(u)$ denoting the distance of the point $u$ to the boundary of $D$. It is then easy to see that the equation takes the form

$$
w_{t}-\left(u^{\alpha} F(t, u, w, D w) w_{u u}+G(t, u, w, D w)\right)=0 .
$$

The coordinate change $\Phi$ transforms the initial data

$$
g_{t}=-\frac{g_{r}^{2}}{\varphi_{r} r^{n-1}}
$$

into

$$
B(w)=w_{t}-\frac{z_{u}}{\left(r_{u}+r_{w} \frac{\partial w}{\partial u}\right) r_{w} \varphi_{r} r^{n-1}}=0
$$

in $\partial_{0} D \times[0, T]$ which is of the form of $H(t, u, w, D w)$. The reader can verify that if $g^{0} \in C_{s}^{k, 2+\gamma}(\Omega)$, then $w^{0} \in C_{s}^{k, 2+\gamma}(D)$. Moreover, the norm $\left\|w^{0}\right\|_{C_{s}^{k, 2+\gamma}(D)}$ can be made arbitrarily small, by choosing the smooth surface $S$ in sufficiently close to the surface $z=g^{0}(r)$.

Since the change of variables $\Phi$ is smooth, the functions $F$ and $G$ will depend smoothly on the variables $(t, u, w, D w)$ as long as $\left|r_{u}\right|>0$ and $\left|r_{w} z_{u}\right|>0$ for all $u \in D \backslash D_{\delta}$. And, the operator

$$
M w=w_{t}-\left(u^{\alpha} F(t, u, w, D w) w_{u u}+G(t, u, w, D w)\right)
$$

satisfies the hypotheses of Theorem 4.4, [20], which indicates the existence of $w$ since the coordinate change $\Phi$ is smooth and the function $w^{0}$ belongs to the space $C_{s}^{k, 2+\gamma}(D)$.

This finishes also the proof of Theorem 2.1.

As a consequence of Theorem 2.1 and Theorem 4.4 at [20], we obtain the following main result. 
Theorem 2.3. Let $\Omega$ be a compact domain in $\mathbb{R}$ and let $g^{0}$ be a function in the space $C_{s}^{k, 2+\gamma}(\Omega)$ for some nonnegative integer $k$ and some number $\gamma$ in $0<\gamma<1$. Assume that $g^{0}=0$ at $\Gamma, g^{0}>0$ in the interior of $\Omega$ so that

$$
\left|D g^{0}(r)\right|+g^{0}(r) \geq c>0 \quad \forall r \in \Omega .
$$

Then, there exists a number $\tau_{k}$, for (GCFP) admits a solution $g$ in $C_{s}^{k, 2+\gamma}\left(\Omega_{t} \times\right.$ $\left.\left[0, \tau_{k}\right]\right)$.

Proof. As we have seen in Theorem 2.1 the coordinate change $\Phi$ converts the given initial free-boundary problem to the initial value problem with fixed boundary

with

$$
\begin{cases}M w & =0 \quad(u, t) \in D \times[0, T] \\ B w & =0 \quad(u, t) \in \partial_{0} D \times[0, T] \\ w(u, 0) & =w^{0}(u) \quad u \in D\end{cases}
$$

$$
\left\{\begin{array}{l}
M w=w_{t}-\left(x^{\alpha} F(t, u, w, D w) w_{u u}+G(t, u, w, D w)\right) \\
B w=H(t, u, w, D w)
\end{array}\right.
$$

and $w^{0} \in C_{s}^{k, 2+\gamma}(D)$. However we have shown that if $T \leq \tau_{k}$, with $\tau_{k}$ sufficiently small depending on $k$ and $c$, the operator $M$ satisfies all the hypotheses of Theorem 2.2, [20]. Therefore, by this theorem the initial value problem for the equation $M w=0$ admits a solution $w \in C_{s}^{k, 2+\gamma}\left(D \times\left[0, \tau_{k}\right]\right)$. If we express the function $w$ in the old coordinates we obtain a solution $z=g(r, t)$ of the given initial free-boundary problem which belongs to the space $C_{s}^{k, 2+\gamma}\left(\Omega \times\left[0, \tau_{k}\right]\right)$. The computations are the same as in the proof of Theorem 2.1.

\section{3. $C^{\infty}$-regularity}

In this final section we will give the proof of Theorem 2.1. It will follow from the next regularity result.

Theorem 2.4. Assume that for some $T>0$ and some number $\gamma$ in $0<\gamma<1$, $g \in C_{s}^{2+\gamma}\left(\Omega_{t} \times[0, T]\right)$ is a solution of (GCFP) so that $|D g(r, t)|+g(r, t) \geq c>$ $0, \forall(r, t) \in \Omega_{t} \times[0, T]$. Then, for any positive integer $k, \frac{\partial^{k} g}{\partial t^{k}} \in C_{s}^{2+\gamma}\left(\Omega_{t} \times(0, T]\right)$ and for any $\tau$ in $0<\tau<T$ we have

$$
\left\|\frac{\partial^{k} g}{\partial t^{k}}\right\|_{C_{s}^{2+\gamma}\left(\Omega_{t} \times[\tau, T]\right)} \leq C_{k}\left(\tau,\left\|g^{0}\right\|_{C_{s}^{2+\gamma}(\Omega)}\right) .
$$

Proof. We begin with the proof of the theorem for $k=1$. Choose a family of smooth regularization $g_{\epsilon}^{0}$ of the initial data $g^{0}$, approximating $g^{0}$, so that each $g_{\epsilon}^{0}$ is strictly positive in the interior of a compact domain $\Omega_{\epsilon}$, with $g_{\epsilon}=0$ at $\Gamma_{\epsilon}$ and $\left|D g_{\epsilon}^{0}(r)\right|+g_{\epsilon}^{0}(r, t) \geq c>0, \forall r \in \Omega_{\epsilon}$. It follows from the results in Section 2 , that we can choose the functions $g_{\epsilon}^{0}$ so that

$$
\left\|g_{\epsilon}^{0}\right\|_{C_{s}^{2+\gamma}\left(\Omega_{\epsilon}\right)} \leq C\left\|g^{0}\right\|_{C_{s}^{2+\gamma}(\Omega)}
$$


with the constant $C$ independent of $\epsilon$ and $g_{\epsilon}^{0} \rightarrow g^{0}$ as $\epsilon \rightarrow 0$ in the sense that

$$
D_{t} g_{\epsilon} \rightarrow D_{t} g
$$

if $i \leq 1$ and

$$
d_{\epsilon}^{\alpha} D_{r}^{2} g_{\epsilon} \rightarrow d_{\epsilon}^{\alpha} D_{r}^{2} g
$$

with $d_{\epsilon}$ denoting the distance to the boundary of $\Omega_{\epsilon}$.

Denote by $g_{\epsilon}$ the solution to $(\mathbf{G C F P})$ as constructed in Theorem 4.4. It follows from the same theorem that there exists a number $\tau_{1}$ in $0<\tau_{1} \leq T$ which is independent of $\epsilon$ such that $g_{\epsilon} \in C_{s}^{1,2+\gamma}\left(\Omega_{\epsilon, t} \times\left[0, \tau_{1}\right]\right.$. Moreover,

$$
\left\|\frac{\partial g_{\epsilon}}{\partial t}\right\|_{C_{s}^{2+\gamma}\left(\Omega_{\epsilon, t} \times\left[0, \tau_{1}\right]\right)} \leq C\left(\left\|g_{\epsilon}^{0}\right\|_{C_{s}^{1,2+\gamma}\left(\Omega_{\epsilon}\right)}\right) .
$$

On the other hand, since $\left\|g_{\epsilon}^{0}\right\|_{C_{s}^{2+\gamma}\left(\Omega_{\epsilon}\right)} \leq C\left\|g^{0}\right\|_{C_{s}^{2+\gamma}(\Omega)}$ the result in Theorem 4.4 implies that

$$
\left\|g_{\epsilon}\right\|_{C_{s}^{2+\gamma}\left(\Omega_{\epsilon, t} \times[0, T]\right)} \leq C\left(\left\|g^{0}\right\|_{C_{s}^{2+\gamma}(\Omega)}\right)
$$

a bound that is independent of $\epsilon$. Therefore, it is easy to see that passing to a subsequence the sequence $g_{\epsilon}$ will converge to $g$, being the unique weak solution of the equation

$$
g_{t}=\frac{1}{I}\left\{\frac{g^{\alpha} g_{r r}}{r^{n-1}}-(n-1) \frac{g^{\alpha} g_{r}}{r^{n}}\right\}
$$

with initial data $g_{r}(r, 0)=g_{r}^{0}$.

Our first goal is to show that for $\tau$ in $0<\tau<\tau_{1}$ we have

$$
\left\|\frac{\partial g_{\epsilon}}{\partial t}\right\|_{C_{s}^{2+\gamma}\left(\Omega_{\epsilon, t} \times\left[\tau, \tau_{1}\right]\right)} \leq C\left(\tau,\left\|g^{0}\right\|_{C_{s}^{2+\gamma}(\Omega)}\right)
$$

with $C=C\left(\left\|g^{0}\right\|_{C_{s}^{2+\gamma}(\Omega)}\right)$ independent of $\epsilon$. For this purpose we will use the local change of variables introduced in Section 2.

For $\epsilon>0$ and $\tau$ in $0<\tau<\tau_{1}$ fixed, we pick a point $P_{0}=r_{0}$ at the free boundary $\partial \Omega_{\epsilon, t} \times\left(\tau, \tau_{1}\right)$. We can assume without loss of generality that $\left(g_{\epsilon}\right)_{r}\left(P_{0}\right)>0,\left(g_{\epsilon}\right)_{t}\left(P_{0}\right)=c$. Then, as we showed, in Section 2, locally around the point $P_{0}$, we can solve the equation $z=g_{\epsilon}(r, t)$ with respect to $r$ yielding to a function $r=h_{\epsilon}(z, t)$ defined on a small box

$$
B_{\eta}=\left\{0 \leq z \leq \eta,-\eta \leq t-t_{0} \leq 0\right\} .
$$

After a moment of thought we realize that the number $\eta$ can be taken to be independent of the particular point $P_{0}$ on the free boundary $\Omega_{\epsilon, t} \times[0, T]$ and independent $\epsilon$. This is because the norms $\left\|g_{\epsilon}\right\|_{C_{s}^{2+\gamma}\left(\Omega_{\epsilon} \times[0, T]\right)}$ are uniformly bounded.

To simplify the notation we will denote for the moment the function $h_{\epsilon}$ by $h$. We have computed in Section 1 that on the set $B_{\eta}$ we have $h_{z}=\frac{1}{g_{r}}$ and therefore $h_{z}(z, t)>0$ if $\eta$ is sufficiently small. Moreover, each $h_{\epsilon}$ satisfies the equation

$$
h_{t}=\frac{1}{J} z^{\alpha} \frac{h_{z z}}{h^{n-1} h_{z}^{2}}+(n-1) \frac{1}{J} \frac{z^{\alpha}}{h^{n}}
$$


for all $(z, t) \in B_{\eta}$. Notice that we can choose $\gamma$ small enough so that $J$ will belong to $C_{s}^{\gamma}$.

Our goal is to establish a $C_{s}^{1,2+\gamma}$ bound for $h=h_{\epsilon}$ in the box $B_{\eta}$ which is independent of $\epsilon$. To do so we will first compute, by differentiating the above equation by $t$, the evolution of the derivatives $h_{t}$ on $B_{\eta}$. Once again the reader may notice that $J$ is depends only on $z$ which has no effect when differentiating the above equation with respect to $t$. Then we will apply the local Schauder estimates. We will establish this bound for the time derivative $w=h_{t}$ which satisfies the same equation, namely $M_{\epsilon, t}(w)=0$, with

$$
\begin{aligned}
M(h) & =h_{t}-z^{\alpha} \frac{1}{J} \frac{h_{z z}}{h^{n-1} h_{z}^{2}}+(n-1) \frac{1}{J} \frac{z^{\alpha}}{h^{n}}=0 \\
M_{\epsilon, t}(w) & =w_{t}-\frac{1}{J}\left\{z^{\alpha} \frac{w_{z z}}{h h_{z}^{2}}+(n-1) z^{\alpha}\left(-\frac{h_{z z}}{h^{n} h_{z}^{2}}+n \frac{1}{h^{n+1}}\right) w\right\} .
\end{aligned}
$$

We conclude from the above that $h=h_{\epsilon}$ satisfies

$$
\left\|\frac{\partial h_{\epsilon}}{\partial t}\right\|_{C_{s}^{1,2+\gamma}\left(B_{\eta / 2}\right)} \leq C\left(\left\|h^{0}\right\|_{C_{s}^{2+\gamma}(\Omega)}\right) .
$$

We can now go back to the original coordinates. The transformation of first and second derivatives via the coordinate change, as computed in Section 2, and our estimates above show that if for some number $\tau$ in $0<\tau<\tau_{1}, A_{\eta, \epsilon}^{\tau}$ denotes the set

$$
A_{\eta, \epsilon}^{\tau}=\left\{(r, t) \in \Omega_{\epsilon} \times\left[\tau, \tau_{1}\right]: g_{\epsilon}(r, t) \leq \eta\right\}
$$

then we have

$$
\left\|\frac{\partial g_{\epsilon}}{\partial t}\right\|_{C_{s}^{2+\gamma}\left(A_{\eta, \epsilon}^{\tau}\right)} \leq C\left(\tau,\left\|g^{0}\right\|_{C_{s}^{2+\gamma}(\Omega)}\right)
$$

The same estimate holds true on the interior set

$$
\Gamma_{\eta, \epsilon}^{\tau}=\left(\Omega_{\epsilon, t} \times\left[\tau, \tau_{1}\right]\right) \backslash A_{\eta, \epsilon}^{\tau}
$$

as it follows from the standard regularity theory of non-degenerate parabolic equations. Therefore we conclude that

$$
\left\|\frac{\partial g_{\epsilon}}{\partial t}\right\|_{C_{s}^{2+\gamma}\left(\Omega_{\epsilon} \times\left[\tau, \tau_{1}\right]\right)} \leq C\left(\tau,\left\|g^{0}\right\|_{C_{s}^{2+\gamma}(\Omega)}\right)
$$

for all $\tau$ in $0<\tau<\tau_{1}$, as desired.

Next we extend each solution $g_{\epsilon}$ so that $g_{\epsilon}(r, t)=0$ for all $r \in \mathbb{R} \backslash \Omega_{\epsilon, t}$, $0 \leq t \leq \tau_{1}$ so that each $g_{\epsilon}$ becomes a weak solution of (GCFP).

The sequence of solutions $\left\{g_{\epsilon}\right\}$ is equicontinuous and uniformly bounded on $R \times\left[0, \tau_{1}\right]$, since

$$
\left\|g_{\epsilon}\right\|_{C_{s}^{2+\gamma}\left(\Omega_{\epsilon} \times\left[0, \tau_{1}\right]\right)} \leq C\left(\left\|g^{0}\right\|_{C_{s}^{2+\gamma}(\Omega)}\right) .
$$

Therefore there exists a subsequence, still denoted by $g_{\epsilon}$ which converges, as $\epsilon \rightarrow 0$, to the given solution $g$ (extended to be equal to zero outside $\Omega_{t} \times\left[0, \tau_{1}\right]$ ). 
The convergence is uniform on compact subsets of $\mathbb{R} \times\left[0, \tau_{1}\right]$. In particular

$$
\operatorname{dist}\left(\Gamma_{\epsilon, t}, \Gamma_{t}\right) \rightarrow 0 \quad \text { as } \epsilon \rightarrow 0
$$

uniformly on $t$ in the interval $0 \leq t \leq \tau_{1}$. However, when restricted on $\Omega_{t} \times$ $\left[\tau, \tau_{1}\right]$, for any $\tau$ in $0<\tau<\tau_{1}$ the convergence is much stronger. Indeed, it follows from the uniform bound

$$
\left\|\frac{\partial g_{\epsilon}}{\partial t}\right\|_{C_{s}^{2+\gamma}\left(\Omega_{\epsilon} \times\left[\tau, \tau_{1}\right]\right)} \leq C\left(\tau,\left\|g^{0}\right\|_{C_{s}^{2+\gamma}(\Omega)}\right)
$$

that there exists a subsequence of $\left\{g_{\epsilon}\right\}$, still denoted by $g_{\epsilon}$, such that for any $(r, t) \in \Omega \times\left[\tau, \tau_{1}\right]$ we have

$$
\left.D_{t}^{i} g_{\epsilon}(r, t) \rightarrow D\right)_{t}^{i} g(r, t)
$$

and

$$
\left(d^{\epsilon}\right)^{\alpha} D_{r}^{2} g_{\epsilon}(r, t) \rightarrow d^{\alpha} D_{r}^{2} g(r, t),
$$

as $\epsilon$ tends to zero, where $d^{\epsilon}$ denotes the distance to the free boundary $\Omega_{\epsilon} \times\left[\tau, \tau_{1}\right]$. It is now easy to check that the solution $\frac{\partial g}{\partial t}$ belongs to the space $C_{s}^{2+\gamma}\left(\Omega_{\epsilon} \times\right.$ $\left.\left(0, \tau_{1}\right]\right)$ and for all $\tau$ in $0<\tau<\tau_{1}$ satisfies the estimate

$$
\left\|\frac{\partial g}{\partial t}\right\|_{C_{s}^{2+\gamma}\left(\Omega_{t} \times\left[\tau, \tau_{1}\right]\right)} \leq C\left(\tau,\left\|g^{0}\right\|_{C_{s}^{2+\gamma}(\Omega)}\right) .
$$

Finally, assume that $N \tau_{1}<T \leq(N+1) \tau_{1}$ for some nonnegative integer $N$. Since

$$
\|g\|_{C_{s}^{2+\gamma}\left(\Omega_{t} \times[0, T]\right)} \leq C\left(\left\|g^{0}\right\|_{C_{s}^{2+\gamma}(\Omega)}\right)
$$

we can repeat the above estimate $N+1$ times to finally conclude that

$$
\|g\|_{C_{s}^{1,2+\gamma}\left(\Omega_{t} \times[\tau, T]\right)} \leq C\left(\tau,\left\|g^{0}\right\|_{C_{s}^{2+\gamma}(\Omega)}\right)
$$

for all $0<\tau<T$, as desired.

This proves the theorem in the case of $k=1$. The case of a general $k$ can be shown via induction by differentiating the local equation

$$
h_{t}-z^{\alpha} \frac{1}{J} \frac{h_{z z}}{h^{n-1} h_{z}^{2}}+(n-1) \frac{1}{J} \frac{z^{\alpha}}{h^{n}}=0
$$

$k$ times and using once more the local Schauder estimate Theorem 4.3.

We finish this section with the proof of Theorem 2.1.

Proof of Theorem 2.1. From Theorem 4.4, there exists a solution $g \in C_{s}^{2+\gamma}$ $\left(\Omega_{t} \times[0, T]\right)$ of the free boundary problem (GCFP) for some number $T>0$. Moreover, since $D g^{0} \neq 0$ at $\partial \Omega$, we can choose the number $T$ so that

$$
|D g(r, t)|+g(r, t) \geq c \quad \forall(r, t) \in \Omega_{t} \times[0, T]
$$

for some $c>0$. But then, it follows from Theorem 5.5 that $g \in C_{s}^{1,2+\gamma}\left(\Omega_{t} \times\right.$ $[0, T])$ for all positive integers. Using the fact that at the free boundary $z=0$ and the coefficients of the equation of $\left(\bar{D}_{r} D_{r}\right)^{k} g \in C_{s}^{2+\gamma}$ and $D_{r}\left(\bar{D}_{r} D_{r}\right)^{k} g \in$ $\tilde{C}_{s}^{2+\gamma}$ for all $k$, we conclude that the free-boundary is smooth. 


\section{Estimates for long time existence}

This section is devoted to the existence up to the focusing time of the nonconvex surface. In order to obtain non-degenerate finite speed of propagation of the free boundary till focusing time, we need to provide the lower bound and the upper bound for $g_{t}$ by estimating $g_{r}$ as in the following two lemmas. First, we can notice the equation (GCFP) is uniformly elliptic in $\Omega_{t}^{(2 k)} \backslash \Omega_{t}^{k}$ for a large $K>0$ since $0<k<g<2 k$ and then $g$ is smooth. Now we choose a large $K$ so that $g_{r}(r, t)>1$ if $g(r, t)=k$. Otherwise from the Harnack inequality, we can find a path on which $g$ goes to infinity while $g_{r} \leq 1$. It is a contradiction.

\section{Lemma 3.1.}

$$
\max _{\Omega_{t}^{k} \times[0, T]}\left|g_{r}(x, t)\right| \leq C \max _{\Omega_{0}^{k}}\left|g_{r}^{0}(x)\right|
$$

in $\Omega_{t}^{k} \times[0, T]$.

Proof. Set $Z(T)=\max _{\Omega_{t}^{k} \times[0, T]} g_{r}$ and assume that the very large maximum is achieved at $\left(r_{o}, t_{o}\right)$.

First $r_{o}$ is not on $\{g=k\}$. When $r_{o}$ is an interior point of $\Omega(g)$, let us consider the evolution of $g_{r}$ :

$$
\begin{aligned}
g_{r, t}= & \frac{1}{I^{n+1}} \frac{g^{\alpha} g_{r r r}}{r^{n-1}}+\frac{1}{I^{n+1}} \frac{\alpha g^{\alpha-1} g_{r} g_{r r}}{r^{n-1}}-\frac{1}{I^{n+1}} \frac{(n-1) g^{\alpha} g_{r r}}{r^{n}} \\
& -\frac{1}{I^{n+1}} \frac{(n-1) \alpha g^{\alpha-1} g_{r}^{2}}{r^{n}}+\frac{1}{I^{n+1}} \frac{n(n-1) g^{\alpha} g_{r}}{r^{n+1}} \\
& -\frac{(n+3) g_{r}}{n I^{n+3}}\left\{\frac{g^{\alpha} g_{r r}}{r^{n-1}}-(n-1) \frac{g^{\alpha} g_{r}}{r^{n}}\right\}(n g)^{2 / n-1} \\
& -(n+1) \frac{2 g^{\frac{1}{n}} g_{r} g_{r r}}{r^{n-1} I^{2 n+4}}-(n+1) \frac{g^{\frac{1}{n}-1} g_{r}^{3}}{n r^{n-1} I^{2 n+4}} \\
& +(n+1)(n-1) \frac{g^{\frac{1}{n}} g_{r}^{2}}{r^{n} I^{2 n+4}}+\frac{2(n+1)(n+2)}{n^{2 / n}} \frac{g^{\frac{3}{n}} g_{r}^{3}}{r^{n-1} I^{2 n+5}} \\
\leq & C(g, r) g_{r} .
\end{aligned}
$$

Then $Z$ will satisfy $Z_{t} \leq C Z$ and then $Z(T) \leq Z(0) e^{C T}$.

Now let us consider the case when $r_{o}$ is on the free boundary $\Gamma$. Then

$$
0 \geq \frac{2 g^{\alpha} g_{r r}}{r_{o}^{n-1}}=g_{t}=-\frac{g_{r}^{2}}{\varphi_{r} r^{n-1}}>0
$$

which is a contradiction. Therefore the maximum will achieved at $t=0$.

\section{Lemma 3.2.}

$$
\min _{\Omega_{t}^{k} \times[0, T]} g_{r} \geq \delta r^{n-1} \min _{\Omega_{0}^{k}}\left|g_{r}^{0}(x)\right|>0
$$

in $\Omega_{t}^{k} \times[0, T]$. 
Proof. Let us consider

$$
Z=\min _{\Omega_{t}^{k} \times[0, T]} e^{-C g^{1-\alpha}} \frac{g_{r}}{r^{n-1}}
$$

and assume that the very small minimum is achieved at $\left(r_{o}, t_{o}\right)$.

Now we are going to show that $Z>\delta_{1}(r)$ that is equivalent to the $g_{r}>\delta(r)$. First $r_{o}$ is not on $\{g=k\}$. When $r_{o}$ is an interior point of $\Omega(g)$, we have

$$
Z_{r}=e^{-C g^{1-\alpha}}\left(\frac{g_{r r}}{r^{n-1}}-\frac{(n-1) g_{r}}{r^{n}}-\frac{C g^{-\alpha} g_{r}^{2}}{r^{n-1}}\right)=0
$$

and

$$
\begin{aligned}
0 & \leq \frac{g^{\alpha} Z_{r r}}{r^{n-1} I^{n+1}}-Z_{t} \\
& =-C(1-\alpha) e^{-C g^{1-\alpha}} g^{-\alpha} r^{1-2 n} g_{t}^{2}\left((n-1) g^{\alpha}+C(1-\alpha) r g_{r}-C g_{r}^{3}\right)<0 .
\end{aligned}
$$

Therefore $r_{o}$ can not be an interior point of $\Omega(g)$. On the boundary, $g_{t}=$ $-\frac{g_{r}^{2}}{\varphi_{r} r^{n-1}}$ so

$$
\begin{aligned}
0 & \leq g^{\alpha} Z_{r}=e^{-C g^{1-\alpha}}\left(\frac{g^{\alpha} g_{r r}}{r^{n-1}}-g^{\alpha} \frac{(n-1) g_{r}}{r^{n}}-\frac{C g_{r}^{2}}{r^{n-1}}\right) \\
& =-e^{-C g^{1-\alpha}}\left(C-\frac{1}{\varphi_{r}}\right) \frac{g_{r}^{2}}{r^{n-1}}<0
\end{aligned}
$$

for $C>\frac{1}{\varphi_{r}}$. Therefore the minimum will achieved at $t=0$.

By Lemma 3.1 and Lemma 3.2, the following estimate of $g_{t}$ guarantees finite speed of propagation of the free boundary.

\section{Lemma 3.3.}

$$
g_{t}<C\left|g^{0}(x)\right|_{C_{s}^{2}\left(\Omega_{0}^{k}\right)}
$$

Proof. Set $Z(T)=\max _{\Omega_{t}^{k} \times[0, T]}\left(\frac{g_{t}}{g_{r}}+\varepsilon t\right)$ and assume that the very large maximum is achieved at $\left(r_{o}, t_{o}\right)$. Then we may assume $g^{\alpha} g_{r r}>\frac{1}{2} Z=\frac{1}{2} g_{t}$ at $\left(r_{o}, t_{o}\right)$. If $t_{o}>0$, then it will be an interior point since

$$
0 \leq g_{t}=-\frac{g_{r}^{2}}{\varphi_{r} r^{n-1}}<C
$$

on the boundary and $g$ is smooth on $\{g=k\}$. First $g_{t}$ satisfies

$$
\begin{aligned}
g_{t t}= & \frac{1}{I^{n+1}}\left\{\frac{g^{\alpha} g_{t, r r}}{r^{n-1}}+\frac{\alpha g_{r r} g^{\alpha-1} g_{t}}{r^{n-1}}\right. \\
& \left.-(n-1) \frac{g^{\alpha} g_{t, r}}{r^{n}}-\alpha(n-1) \frac{g^{\alpha-1} g_{t} g_{r}}{r^{n}}\right\} \\
& -\frac{(n+3) g_{t}}{n I^{n+3}}\left\{\frac{g^{\alpha} g_{r r}}{r^{n-1}}-(n-1) \frac{g^{\alpha} g_{r}}{r^{n}}\right\}(n g)^{2 / n-1}
\end{aligned}
$$




$$
\begin{aligned}
& -(n+1) \frac{2 g^{\frac{1}{n}} g_{r} g_{t r}}{r^{n-1} I^{2 n+4}}-(n+1) \frac{g^{\frac{1}{n}-1} g_{r}^{2} g_{t}}{n r^{n-1} I^{2 n+4}} \\
& +\frac{2(n+1)(n+2)}{n^{2 / n}} \frac{g^{\frac{3}{n}} g_{r}^{2} g_{t}}{r^{n-1} I^{2 n+5}} .
\end{aligned}
$$

At the interior point $\left(r_{o}, t_{o}\right)$, we have

$$
Z_{r r}=\left(\frac{g_{t}}{g_{r}}\right)_{r r} \geq 0, \quad Z_{r}=\left(\frac{g_{t}}{g_{r}}\right)_{r}=0 .
$$

Then (3.1) and (3.2) gives us the following contradiction at $\left(r_{o}, t_{o}\right)$ :

$$
\begin{aligned}
0 \geq Z_{t}= & \left(\frac{g_{t}}{g_{r}}\right)_{t}-\varepsilon \\
= & \frac{g_{r} g_{t t}-g_{t} g_{r t}}{g_{r}^{2}} \\
= & \frac{g^{\alpha}}{r^{n-1} I^{n+1}}\left(\frac{g_{t}}{g_{r}}\right)_{r r}+\frac{2 g^{\alpha} g_{r r}}{r^{n-1} I^{n+1} g_{r}}\left(\frac{g_{t}}{g_{r}}\right)_{r} \\
& -(n-1) \frac{g^{\alpha}}{r^{n} I^{n+1}}\left(\frac{g_{t}}{g_{r}}\right)_{r}-(n+1) \frac{2 g^{\frac{1}{n}} g_{r}}{r^{n-1} I^{2 n+4}}\left(\frac{g_{t}}{g_{r}}\right)_{r}+\varepsilon \\
\geq & +\varepsilon>0 .
\end{aligned}
$$

Therefore the large maximum will be achieved at the initial time.

On the other hand, the next lemma guarantees non-degenerate speed at the free boundary up to the focusing time.

\section{Lemma 3.4.}

$$
\min _{\Omega_{t}^{k} \times[0, T]} g_{r r}(x, t) \geq-C\left(\max _{\Omega_{0}^{k}}\left|g_{r r}^{0}(x)\right|+C_{1}(K)\right)
$$

in $\Omega_{t}^{k} \times[0, T]$.

Proof. Set $Z(T)=\min _{\Omega_{t}^{k} \times[0, T]} g_{r r}$ and assume that the very large negative minimum is achieved at $\left(r_{o}, t_{o}\right)$. If $\left(r_{o}, t_{o}\right)$ is an interior point, we have a contradiction since, at $\left(r_{o}, t_{o}\right)$,

$$
\begin{aligned}
0 \geq & g_{r r, t} \\
= & \frac{1}{I^{n+1}} \frac{g^{\alpha} g_{r r r r}}{r^{n-1}}+2\left(\frac{1}{I^{n+1}} \frac{g^{\alpha}}{r^{n-1}}\right)_{r} g_{r r r} \\
& +\left(1+(n g)^{2 / n}\right)^{1-\frac{n+5}{2}} r^{1-n}\left(\alpha g^{\alpha-1}+C_{1} g^{\alpha-1+\frac{2}{n}}\right) g_{r r}^{2} \\
& -\frac{1}{n^{2}} g^{-2+\alpha}\left(1+(n g)^{2 / n}\right)^{\frac{1}{2}(-5-n)} r^{-1-n}\left(n^{2}(1-\alpha) \alpha r^{2} g_{r}^{2}+C_{2} g^{\frac{2}{n}}\right) g_{r r} \\
& +g^{-2+\alpha}\left(1+(n g)^{2 / n}\right)^{-\frac{1}{2}(5+n)}(n-1) \alpha(1-\alpha) r^{-n} g_{r}^{3} \\
& +C_{3} g^{-2+\alpha+2 / n}+C_{4} g^{1 / n-2}>0
\end{aligned}
$$


for some $C_{3}, C_{4} \geq 0$. On $\partial\{g>0\}$,

$$
\lim _{r \rightarrow \gamma\left(t_{o}\right)} g^{\alpha} g_{r r}=-\frac{g_{r}^{2}}{\varphi_{r} r_{0}^{n-1}}>0 .
$$

Hence the negavitive infimum of $g_{r r}$ doesn't happens on $\partial\{g>0\}$. And since $g$ is smooth on $\{g=k\}$, there is uniform lower bound of $g_{r r}$ depending on $K$.

Lemma 3.5. There exist $0<\delta<c_{o}<A<\infty$ such that

$$
0<\delta(T) \leq\left(t+c_{0}\right) \frac{g_{t}(x, t)}{g_{r}}+A g(x, t)
$$

for $(x, t) \in \Omega_{t}^{k} \times[0, T]$.

Proof. There is $A>0$ such that $g_{t}(x, t)+A K>1>0$. Set $Z(T)=$ $\min _{\Omega_{t}^{k} \times[0, T]}\left(\left(t+c_{0}\right) \frac{g_{t}(x, t)}{g_{r}}+A g(x, t)\right)=\delta$ and assume that the very small positive minimum, $\delta$, is achieved at $\left(r_{o}, t_{o}\right)$. On the boundary,

$$
0<\delta \leq-\frac{g_{r}}{\varphi_{r} r^{n-1}}=\frac{g_{t}}{g_{r}}
$$

for a small $\delta>0$. $r_{o}$ is not on $\{g=k\}$ from the choice of $A$. When $\left(r_{o}, t_{o}\right)$ is an interior point, we have, at $\left(r_{o}, t_{o}\right)$,

and the following contradiction:

$$
Z_{r r}=\left(\frac{g_{t}}{g_{r}}\right)_{r r}+A g_{r r} \geq 0, \quad Z_{r}=\left(\frac{g_{t}}{g_{r}}\right)_{r}+A g_{r}=0
$$

$$
\begin{aligned}
0 \geq Z_{t}= & \frac{g_{r} g_{t t}-g_{t} g_{r t}}{g_{r}^{2}} \\
= & \frac{g^{\alpha}}{r^{n-1} I^{n+1}} Z_{r r}+\frac{2 g^{\alpha} g_{r r}}{r^{n-1} I^{n+1} g_{r}} Z_{r} \\
& -(n-1) \frac{g^{\alpha}}{r^{n} I^{n+1}} Z_{r}-(n+1) \frac{2 g^{\frac{1}{n}} g_{r}}{r^{n-1} I^{2 n+4}} Z_{r} \\
& +(n+1) \frac{A g^{\frac{1}{n}} g_{r}^{2}}{r^{n-1} I^{2 n+4}}>0 .
\end{aligned}
$$

Lemma 3.6.

$$
0<\delta \leq g^{\alpha} g_{r r} \leq C
$$

in a uniform neighborhood of $\Gamma(g)$.

Proof of Theorem 1.2. Let $S_{t}^{k}=\left\{(x, y, f(x, y, t)) \in \Sigma(t) \mid(x, y) \in \Omega_{t}^{k}\right\}$ be the graph of $f$ on $\Omega^{k}(t)$ and $\Sigma^{k}=\Sigma \backslash S^{k}$. Set $h_{i j}$ a second fundamental form of $\Sigma$ at $P$ and $\left(h^{-1}\right)^{i j}$ the inverse of $h_{i j}$.

The Gauss curvature $K$ in the $\Sigma^{k / 2}$ satisfies

$$
\frac{\partial}{\partial t} K=K\left(h^{-1}\right)^{i j} \nabla_{i} \nabla_{j} K+K^{2} H
$$

[1], and then a minimum principle which means that the minimum of $K$ happens either on initial surface $\Sigma^{k / 2}(0)$ or the boundary $\partial \Sigma^{k / 2}(t)$ for $t \in\left[0, T_{c}\right]$. 
On the other hand, $K=\frac{f_{r}^{n-1} f_{r r}}{r^{n-1} I^{n}}=\frac{g_{r}}{r^{n-1} I^{n}}$ is bounded above and below on $\partial \Sigma^{k / 2}$. Therefore $K$ will be bounded below by a uniform constant $\delta_{0}$.

The Harnack estimate from [4] says that if $z \in S^{n-1}$ and $P(z, t) \in \Sigma$ is the point with $-N(P(z, t))=z$ for each $t$, then

$$
\frac{d}{d t}\left(t^{2 / 3} K(p(z, t), t) \geq 0\right.
$$

which implies that there is $0<\delta_{0}$ such that $K(P)>\delta_{0}$ for a point in the strictly convex part $\Sigma^{k}$. From the same argument as Proposition 7 in [1], the Gauss curvature will be bounded in $\left[0, T_{c}\right]$. Since the mean curvature $H$ is also bounded on $\partial \Sigma^{k / 2}(t)$ for $t \in\left[0, T_{c}\right], H$ will be also bounded in $\Sigma^{k / 2}(t)$. Now the principle curvatures, $\lambda_{1}, \ldots, \lambda_{n}$, will be uniformly bounded, the diffusion coefficient of the linearized equation will be uniformly elliptic, i.e., there is $\Lambda>0$ such that

$$
\frac{1}{\Lambda}<\lambda_{i}<\Lambda \text { for } i=1, \ldots, n
$$

and

$$
\frac{1}{\Lambda}|\xi|^{2}<K\left(h^{-1}\right)^{i j} \xi_{i} \xi_{j}<\Lambda|\xi|^{2}
$$

Then the regularity theory for uniformly elliptic and parabolic theory, [19], $\Sigma^{k}$ will be smooth.

With the smoothness of $\Sigma^{k}$, lemmas in this section, and Theorem 2.2 and Theorems 4.3, 4.4 at [20], the function $g$ will be smooth up to the free boundary for all time $0<t<T_{c}$, with the focusing time $T_{c}$ of the non-convex surface. And the free boundary will be smooth.

Acknowledgement. Ki-Ahm Lee was supported by the Korea Research Foundation Grant funded by the Korean Government(MOEHRD, Basic Research Promotion Fund)(KRF-2008-314-C00023).

\section{References}

[1] B. Andrews, Gauss curvature flow: The fate of the rolling stones, Invent. Math. 138 (1999), no. 1, 151-161.

[2] D. Chopp, L. C. Evans, and H. Ishii, Waiting time effects for Gauss curvature flow, Indiana Univ. Math. J. 48 (1999), no. 1, 311-334.

[3] B. Chow, Deforming convex hypersurfaces by the nth root of the Gaussian curvature, J. Differential Geom. 22 (1985), no. 1, 117-138.

[4] - On Harnack's inequality and entropy for the Gaussian curvature flow, Comm. Pure Appl. Math. 44 (1991), no. 4, 469-483.

[5] P. Daskalopoulos and R. Hamilton, The free boundary in the Gauss curvature flow with flat sides, J. Reine Angew. Math. 510 (1999), 187-227.

[6] - The free boundary for the n-dimensional porous medium equation, Internat. Math. Res. Notices 1997 (1997), no. 17, 817-831.

[7] L Regularity of the free boundary for the porous medium equation, J. Amer. Math. Soc. 11 (1998), no. 4, 899-965.

[8] — $C^{\infty}$-regularity of the interface of the evolution pp-Laplacian equation, Math. Res. Lett. 5 (1998), no. 5, 685-701. 
[9] P. Daskalopoulos, R. Hamilton, and K. Lee, All time $C^{\infty}$-regularity of the interface in degenerate diffusion: a geometric approach, Duke Math. J. 108 (2001), no. 2, 295-327.

[10] P. Daskalopoulos and K. Lee Free-Boundary Regularity on the Focusing Problem for the Gauss Curvature Flow with Flat sides, Math. Z. 237 (2001), no. 4, 847-874.

[11] _ Worn stones with flat sides all time regularity of the interface, Invent. Math. 156 (2004), no. 3, 445-493.

[12] _ Hölder regularity of solutions of degenerate elliptic and parabolic equations, J. Funct. Anal. 201 (2003), no. 2, 341-379.

[13] P. Daskalopoulos and E. Rhee Free-boundary regularity for generalized porous medium equations, Commun. Pure Appl. Anal. 2 (2003), no. 4, 481-494.

[14] W. Firey, Shapes of worn stones, Mathematica 21 (1974), 1-11.

[15] R. Hamilton, Worn stones with flat sides, A tribute to Ilya Bakelman (College Station, TX, 1993), 69-78, Discourses Math. Appl., 3, Texas A \& M Univ., College Station, TX, 1994.

[16] H. Ishii and T. Mikami, A mathematical model of the wearing process of a nonconvex stone, SIAM J. Math. Anal. 33 (2001), no. 4, 860-876.

[17] A level set approach to the wearing process of a nonconvex stone, Calc. Var. Partial Differential Equations 19 (2004), no. 1, 53-93.

[18] N. V. Krylov and N. V. Safonov, A property of the solutions of parabolic equations with measurable coefficients, Izv. Akad. Nauk SSSR Ser. Mat. 44 (1980), no. 1, 161-175.

[19] G. M. Lieberman, Second Order Parabolic Differential Equations, World Scientific Publishing Co., Inc., River Edge, NJ, 1996.

[20] K.-A. Lee and E. Rhee, Rolling Stones with nonconvex sides II: All time regularity of Interface and surface, preprint.

[21] K. Tso, Deforming a hypersurface by its gauss-Kronecker curvature, Comm. Pure Appl. Math. 38 (1985), no. 6, 867-882.

[22] L. Wang, On the regularity theory of fully nonlinear parabolic equations I, Comm. Pure Appl. Math. 45 (1992), no. 1, 27-76.

[23] Appl. Math. 45 (1992), no. 2, 141-178.

KI-AHM LEE

Department of Mathematics

Seoul National University

SEOUl 151-747, KoreA

E-mail address: kiahm@math.snu.ac.kr

Eunjai RheE

Department of Mathematics

Seoul National University

SEOUL 151-747, KoreA

E-mail address: ejvrhee@yahoo.com 